\title{
State Detection of Bond Wires in IGBT Modules using Eddy Current Pulsed Thermography
}

\author{
Kongjing Li, Member, IEEE, Gui Yun Tian, Senior Member, IEEE, Liang Cheng, Student Member, IEEE, \\ Aijun Yin, Member, IEEE, Wenping Cao, Senior Member, IEEE and Stuart Crichton, Student Member, IEEE
}

\begin{abstract}
Insulated gate bipolar transistor (IGBT) modules are important safety critical components in electrical power systems. Bond wire lift-off, a plastic deformation between wire bond and adjacent layers of a device caused by repeated power/thermal cycles, is the most common failure mechanism in IGBT modules. For the early detection and characterisation of such failures, it is important to constantly detect or monitor the health state of IGBT modules, and the state of bond wires in particular. This paper introduces eddy current pulsed thermography (ECPT), a non-destructive evaluation (NDE) technique, for the state detection and characterisation of bond wire lift-off in IGBT modules. After the introduction of the experimental ECPT system, numerical simulation work is reported. The presented simulations are based on the 3-D electromagnetic-thermal coupling finite-element-method (FEM) and analyse transient temperature distribution within the bond wires. This paper illustrates the thermal patterns of bond wires using inductive heating with different wire statuses (lifted-off or well bonded) under two excitation conditions: non-uniform and uniform magnetic field excitations. Experimental results show that uniform excitation of healthy bonding wires, using a Helmholtz coil, provides the same eddy currents on each, whilst different eddy currents are seen on faulty wires. Both experimental and numerical results show that ECPT can be used for the detection and characterisation of bond wires in power semiconductors through the analysis of the transient heating patterns of the wires.

The main impact of this work is that it is the first time electromagnetic induction thermography, so-called ECPT, has been employed on power/electronics devices. Because of its capability of contactless inspection of multiple wires in a single pass, and as such it opens a wide field of investigation in power/electronics devices for failure detection, performance characterisation and health monitoring.
\end{abstract}

Index Terms-Power electronic modules, Insulated gate bipolar transistor (IGBT), Bond wire lift-off, Eddy current pulsed thermography, Non-destructive evaluation.

Manuscript received August 13, 2013; revised September 17, 2013; accepted October 16, 2013. Date of current version October 22, 2013. Recommended for publication by Associate Editor Lindsay Sorensen. This work was partially supported by Europe Health Monitoring of Offshore Wind Farms (HEMOW).

Kongjing Li, L. Cheng, W. Cao and S. Crichton are with the School of Electrical and Electronic Engineering, Newcastle University, Newcastle upon Tyne, NE1 7RU, U.K. (e-mail:k.li3@ncl.ac.uk; liang.cheng@ncl.ac.uk; s.o.j.crichton@ncl.ac.uk).

G. Y. Tian is with the School of Automation Engineering, University of Electronic Science and Technology of China, Chengdu 611731, China, and also with the School of Electrical and Electronic Engineering, Newcastle University, Newcastle upon Tyne NE1 7RU, U.K. (e-mail:g.y.tian@ncl.ac.uk).

A. Yin is with the State Key Laboratory of Mechanical Transmission, College of Mechanical Engineering, Chongqing University, Chongqing 400044, China, and also with the School of Electrical and Electronic Engineering, Newcastle University, Newcastle upon Tyne NE1 7RU, U.K. (e-mail: aijun.yin@cqu.edu.cn)

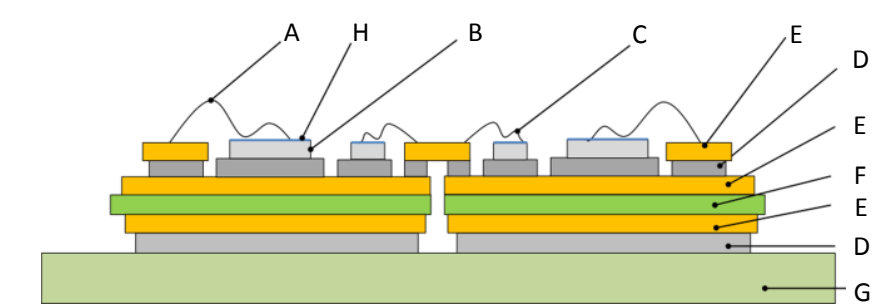

Fig. 1 IGBT structure; a: bond wire, b: IGBT, c: diode, d: solder, e: copper metallisation, f: ceramic substrate, g: base plate, h: aluminium metallisation

\section{INTRODUCTION}

$\mathrm{E}$ LECTRICAL energy converters are widely used in renewable energy and power network domains for motor electrical energy converters are widely used in renewable energy and power network domains for motor control and energy conversions. Failures of these converters can cause catastrophic accidents and result in high penalties and maintenance costs. At present a growing number of these conversion devices are being utilised in human transportation; such as within the aeronautical, automotive and railway traction industries [1]. These transport applications have significant safety requirements. Thus, the reliability of power converters is getting an increasing level of attention from manufacturers. As core components of a power converter, IGBT power modules have high current and voltage ratings and have the strength of being able to handle short-circuits for more than $10 \mu \mathrm{s}$.

Although IGBTs are durable, they have been widely reported to be the most fragile device in converters [2]. IGBTs suffer thermal-mechanical stresses from thermal and power cycling. For this reason, there will be a heavy current flow into any IGBT devices immediately after any form of overload [3]. The progressive failure mechanisms of power modules can be categorised into two groups; chip-related failure mechanisms and package-related failure mechanisms. Chip-related failures often occur without any prior warning, and as such they are hard to monitor and predict, and always result in the destruction of the device. For the monitoring of IGBT device health state, package-related failures are, as such, both more important and practical in terms of detection. These types of failures are driven by thermo-mechanical stresses and often interact with failure mechanisms associated with the chip; as an example problems such as electrical overstress can lead to damage to wires and the die, resulting in a short circuit and failure [4].

IGBT modules have a multi-layered structure consisting of different materials, as shown in Fig. 1. Common failures of a power module are due to the temperature swings caused by power cycling. Adhesion issues and cracks are induced by coefficient mismatches between the thermal expansions (CTEs) 


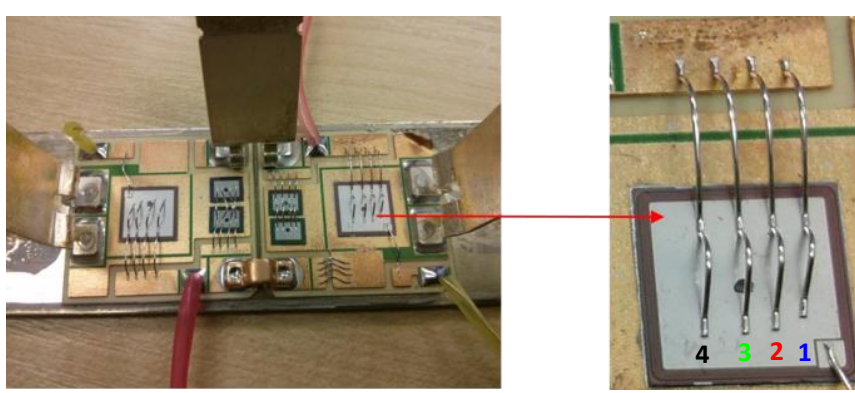

Fig. 2 (a) IGBT configuration, (b) Bond wires on IGBT die

of different adjacently bonded materials under these temperature swings. The main failure mechanisms of IGBTs include bond wire fatigue, resulting in bond wire lift-off and heel cracking; the reconstruction of aluminium metallisation; interconnection corrosion; solder fatigue and solder voids [5]. Some of these failure mechanisms are introduced in the process of manufacture, Li has deeply investigated the microstructural characteristics and interface mechanisms in electronics devices packaging [6-8]. This previous research has shown that non-optimised ultrasonic bonding processes can damage bond wires by producing excessive strains on the bonds. These strains may initiate early stage failures like bond wire lift-off and bond wire heel cracking [4]. The ageing modes of IGBT modules in high-temperature power cycling have also been investigated $[9,10]$. It has been proven that the ageing of bond wires and solder fatigue are the most significant failure mechanisms in IGBT modules [2, 5, 11-14]. To monitor one of the most important failure mechanisms, bond wire failure, this paper introduces an eddy current pulsed thermography (ECPT) system [15-17] for bond wire health state detection and characterisation.

The rest of the paper will be organised as follows: the second section describes the failure mechanism of bond wire lift-off, improvements of packaging technologies and detection methods for bond wire lift-off. An ECPT system and sample descriptions are illustrated in the third section. Simulation work is reported in the fourth section. The experimental set up, experimental results and analyses are reported in the fifth section. Conclusions and future work are then delivered.

\section{BOND WIRE LIFT-OFF AND DETECTION}

Bond wire failures occur predominantly due to fatigue caused by thermo-mechanical shear stresses induced at the bond wire/chip die interface. These stresses are caused by differences in CTEs between $\mathrm{Al}$ and $\mathrm{Si}$ under temperature swings, and/ or by repeated wire loop flexure due to power cycling [5, 9]. With a better understanding of failure mechanisms and continuously developing process technologies, the reliability of power modules has been improved greatly in recent years. For instance, the average failure rate of power modules in traction applications dropped to 20 failures in time (FITs) in 2000 from 1000 FITs in 1995 , where 1 FIT $=1$ failure per 109 device-hours [18]. In addition to this, improved packaging technologies have also been developed. For example, Semikron SkiN is using flexible PCBs to replace $\mathrm{Al}$ wire-bonding to achieve 70 times better power cycling performance. Also, Infineon XT introduced the mass production of copper wire-bonding and transient liquid phase

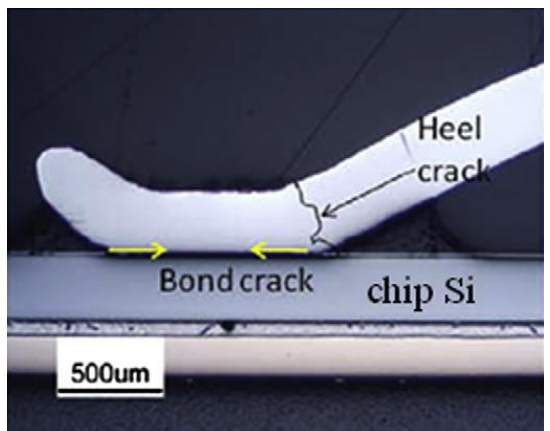

Fig. 3 Lift-off and Heel crack mechanism of a wire bonding [19].

bonding (TLPB) in power modules to improve performance of both die connection and die attachment.

However, in general, the new packaging technologies are of higher cost than traditional Al wire-bonding. Al wire-bonding has been a popular connection solution for over 40 years, it has been a historical method employed since the beginning of microelectronics packaging. Al wire-bonding is a cheap, fast, easy process and the required equipment is widely available. It has been employed in most power electronic devices in the industry, and so the introduction of state monitoring and defect detection methods for them is of great importance. A traditional Al wire-bonded IGBT is shown in Fig. 2, this is the one used in experimental studies. Since the bond lift-off is the most frequent bond wire failure that occurs in IGBT modules, it is the main focus of this paper. Such failures belong to the package-related failure mechanism grouping.

The difference in strains between the silicon chip and aluminium bond wire causes shear stress at their interface. The shear stress is dependent upon the magnitude of temperature swings, $\Delta \mathrm{T}$, encountered during routine power cycling. This is illustrated in Fig. 3 [19]. The resulting cracks are both initiated and propagated by the temperature swings induced by normal operational power cycling. Cracks propagate from both ends of the bond wedge towards the centre along the small grain boundaries of aluminium wires. When the cracks meet at the centre, the bond wire lifts off. Once a bond wire lift-off occurs, the current through other wires will immediately increase. When the current is raised to the point of bond wire rupture, a large amount of energy is released from the bond wires to the soft gel, which surround the wires for electrical and humidity isolation, at this point. The state of the soft gel will change to that of a fluid and then to plasma because of this released thermal energy. At the same time any surrounding wires will fail due to the excessive current level now flowing through them. If the device current is uninterrupted, the module will fail. If the fault continues, it could result in the transferal of all the energy of the power structure bus capacitance to the failure device, which will cause catastrophic damage to the power electronic converter [20]. To prevent such problems, a number of research projects, for the monitoring of bond wire status, have been launched around the world. Some of them are briefly introduced here.

The failure of bond wires changes the distribution of current or the contact resistance, and impacts the gate equivalent capacitance and parasitic inductance of an IGBT module. These physical parameter changes engender effects on external characteristics. Bond wire lift-off could be traced by monitoring VCE since the effective resistance of IGBT 
increases with bond wire lift-off, which initiate drifts in the forward voltage. Furthermore VCE increases as more bond wires lift-off. This method has gained popularity within the research field [9, 21-26]. Besides VCE, other electrical characteristics can also be affected by bond wire lift-off, and as such these characteristics could also be used as precursor parameters to evaluate bond wire lift-off. Methods of gate voltage VG behaviour monitoring [12, 20, 27, 28] and gate current IG measurement [29-31] have also been researched recently. Precision measurement of resistances can monitor bond wire lift-off, as increases in conductive resistance is the direct impact of the failure [32-34]. By monitoring the capacitance of the flying capacitor Css, one of the major origins of failure in IGBT, voltage unbalance can be detected [35]. Furthermore, performance evaluation and loss calculations for new device types, such as $\mathrm{SiC}$, could be achieved by monitoring multiple electrical parameters [36].

However, although such research continues to develop, there are always some innate shortcomings. Changes in external electrical parameters may be caused by other failure mechanisms, such as interconnection corrosions, and metallisation reconstructions amongst others. More importantly, even though most of these methods claim to be able to detect bond wire lift-off during converter operation, there are significant challenges to their implementation with regards to monitoring for such failures, such as varied stress conditions and the difficulties of measuring signals accurately [26]. Last but not least, all of the methods of electrical parameter detection used on bond wire lift-off are in contact with the device under test conditions (DUT). As such sub-circuits to provide electrical sources or external/internal sensors to measure the changes are always required.

Microscopy techniques such like SEM are often used offline to determine the exact cause of bond wire failures [4]. However it is time consuming to determine bond wire failures using SEM, since some modern IGBT modules contain up to 800 bond wires [5].

Nowadays, the concept of non-destructive evaluation (NDE) is attracting more attention by researchers in the electrical field. Traditionally, NDE methods have been employed for the testing of mechanical materials. Recently, an increasing number of institutes have been introducing NDE technology and methods for the inspection of electronic components. A non-destructive closed loop tester was illustrated in [37], the test circuit is based on a typical double pulse clamped inductive load. The circuit sensing voltage collapse (dv/dt) is used to monitor for bond wires failures. The laser ultrasound technique is a popular NDE technology for the testing of complex geometric shapes. Ume has applied it to detect flip-chip solder joint cracks. Solder bump fatigue cracks can be detected efficiently by using such technology [38-40]. X-ray [41] and acoustic methods [42] have also been applied to electronic components and tested widely. However, the laser ultrasound technique is a contact testing method, X-rays are harmful to the human body, and the acoustic methods need high frequency sound waves to get a sufficient resolution. Infrared (IR) cameras have been widely used to monitor temperature distributions across IGBTs; one non-destructive testing technology, lock-in thermography (LIT), has been broadly developed to detect failures on electronic devices. This paper
[43], shows that LIT can be used to determine localised failure spots of UIS failure on IGBTs. The main disadvantage of LIT is the requirement of employing an appropriate thermal wave frequency, otherwise some of the defects cannot be detected. IR cameras have also recently been used to perform thermal mapping of IGBT cross sections [44]. This study shows the feasibility of a method to characterise electrical and thermal distributions in vertical sections with the aid of a high resolution IR camera.

In this paper an advanced NDE technology, ECPT, is applied to detect bond wire lift-off. The major advantage of ECPT over other detection techniques is there is no contact with the inspected sample and there is potential to rapidly inspect a relatively large area within a short amount of time. For this reason, ECPT has been proposed in this paper for rapid detection of bond wire failure in multiple wires. ECPT systems have been employed for the inspection of mechanical systems, such as for crack detection in metallic materials [45], surface crack detection in ferrite and austenitic steel components [46], the estimation of electrical and thermal properties of isotropic materials [47], rail track inspection [15] and surface crack detection for carbon fibre reinforced plastic materials (CFRP) $[48,49]$. In this paper, bond wire lift-off is simulated by manually cutting the wire bond of the IGBT sample. ECPT can detect failures by analysing the thermal patterns of the bond wires. Failures are 'highlighted' for visualisation from the thermal images. The various thermal patterns and transient temperature responses are analysed to identify the failures.

\section{EDDY CURRENT PULSED THERMOGRAPHY ON IGBT}

This section provides a theoretical introduction to the ECPT system, experimental setup and illustrations for excitation coils. Two different excitation conditions have been adopted for non-uniform magnetic field excitation (NMF) and uniform magnetic field (UMF) excitation respectively. NMF excitations are used in [11, 34-36], as the failures generated in regular geometrical conductive materials are normally fatigue cracks. However, in the IGBT power modules, there are hundreds of wires which form complex 3D geometrical shapes. As such it is worth investigating if the NMF excitation is sufficient to achieve the detection of bond wire failure and if it cannot, UMF excitation would be required.

\section{A. Theoretical Considerations}

The ECPT technique employs a magnetic field as excitation, the excitation sources are electric currents. The electromagnetic phenomena in a closed system, e.g. ECPT system, can be investigated on a macroscopic level when the Maxwell's equations are solved in conjunction with proper boundary conditions and constitutive representation of each material property. For the ECPT used in this paper, the frequency is set at $145 \mathrm{kHz}$ at which the wavelength of the electromagnetic wave is much larger than the dimension of the system. A conductive material is then inductively heating by the eddy current. The generated resistive heat $Q$ is proportional to the square of the eddy current density $J_{s}$ or electric field intensity $E$. The relationship between $Q, J_{s}$ and $E$ is governed by the following equation, where $\sigma$ is conductivity. 


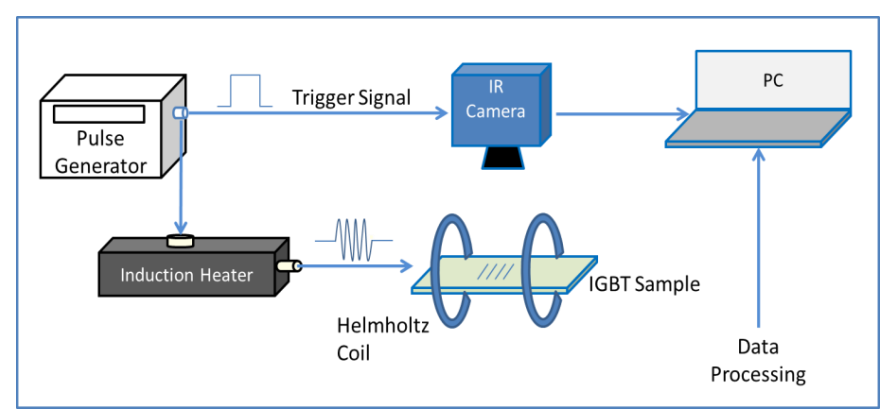

Fig. 4ECPT system diagram

$$
\mathrm{Q}=\frac{1}{\sigma}\left|\mathrm{J}_{\mathrm{s}}\right|^{2}=\frac{1}{\sigma}|\sigma \mathrm{E}|^{2}
$$

The heat diffusion equation of a specimen caused by a Joule heating source $Q$ is governed by

$$
\rho \mathrm{C}_{\mathrm{p}} \frac{\partial \mathrm{T}}{\partial t}-\nabla(k \nabla T)=Q
$$

Where $\rho, C_{p}, k$ are density, heat capacity and thermal conductivity respectively.

\section{B. ECPT systems}

The ECPT system diagram is illustrated in Fig. 4. In the system, a high-frequency and high-power signal generator provides high power currents for induction heating. The infrared (IR) camera and induction heater are controlled by a square pulse signal from the same pulse generator. The pulse is used to both trigger the IR camera to record the thermal videos, and to switch on the induction heater at the same time. Recorded thermal videos are then transmitted to a PC for visualisation and post-processing, including defect identification and electrical/thermal property evaluation.

Fig. 5, which was taken at Newcastle University, shows an Easyheat 224 from Cheltenham Induction Heating which is used for coil excitation. The Easyheat has a maximum excitation power of $2.4 \mathrm{~kW}$, a maximum current of $400 A_{r m s}$ and an excitation frequency range of $140 \mathrm{kHz}-400 \mathrm{kHz}$ (values of $300 A_{r m s}$ and $145 \mathrm{kHz}$ are used in the experiments).

In general, high excitation frequencies will lead to a high thermal contrast (or high temperature rise). The time domain information will allow the derivation of defect profile information. The system has a quoted rise time (from the start of the heating period to full power) of $5 \mathrm{~ms}$, which was verified experimentally. Water cooling of the coil is implemented to counteract direct heating of the coil. The SC655sc is a FLIR IR camera and has an uncooled detector with a maximum frame size of $640 \times 120$ on a $7.5-14.0 \mu \mathrm{m}$ InSb detector. The camera has a sensitivity of $<50 \mathrm{mK}$ and a maximum full frame rate of $50 \mathrm{~Hz}$. The maximum $200 \mathrm{~Hz}$ frame rate provides one frame every $5 \mathrm{~ms}$, with the option to increase the frame rate with windowing of the image, the frame rate is set as $200 \mathrm{~Hz}$ in the experiments.

\section{IGBT Samples}

The IGBT samples used in the experiment are IGBT Power modules with a collector current of 50 A $\max$ and

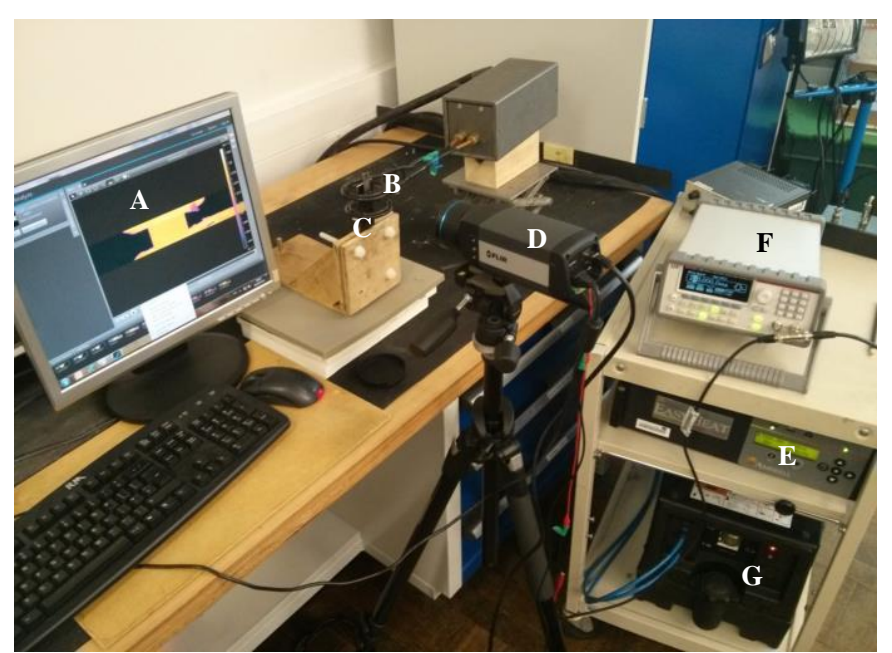

Fig. 5 System configurations, a: PC, b: Excitation coil, c: IGBT sample, d: IR camera, e: Induction heater, f: Pulse generator, g: Cooling system

collector-emitter voltage of $1200 \mathrm{~V}$ max. Each has two IGBTs in a one-package module. The case of the IGBT is opened and no silicone gel exists to enable IR camera recording. As shown in Fig. 2, four bond wires connect the IGBT die to the copper pad, which leads to the outer emitter terminal. Bond wires are made of high purity aluminium $(99.999 \%)$ with diameters of $300 \mu \mathrm{m}$, the wire length is $12 \mathrm{~mm}$ and maximum wire loop height is $2 \mathrm{~mm}$. To reduce the emissivity effect of the bond wires, and the aluminium metallisation of IGBT die to infrared camera, the sample is painted in matte black paint as shown in Fig. 6. After painting, the emissivity value is about 0.95 , a figure which is also adopted in the simulation. To simulate bond wire lift-off, wire 2 in Fig. 2 has been cut from the heel.

As shown in Fig. 5, one $200 \mathrm{~ms}$ heating time followed by $800 \mathrm{~ms}$ cooling time is applied, the acquisition rate is $200 \mathrm{fps}$. However, in real applications, it may be unnecessary to use such a high-end camera. Due to the complex 3-D structure of the IGBT, excitations using the circular and Helmholtz coils were also employed to generate NMF and UMF excitations for the investigation.

As IGBT bond wires exhibit 3-D directional conductivity, dependent on the wire orientation in the IGBT, different coil orientations exert a large impact on experimental results. The distribution of eddy currents generated in the wires is dependent on the orientation of wires to the magnetic field. To maximise the eddy currents induced on the bond wires, the excitation fields of the coils should intersect the wires from the side; thereby inducing currents to flow along them. For this reason, coils are positioned as shown in Fig. 7.

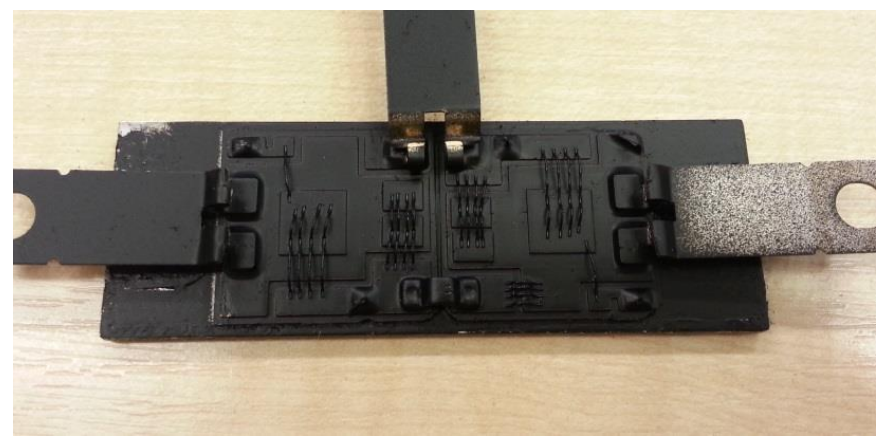

Fig. 6 To overcome the emissivity variation, IGBT sample covered with black matt paint prepared for thermal recording by IR camera. 


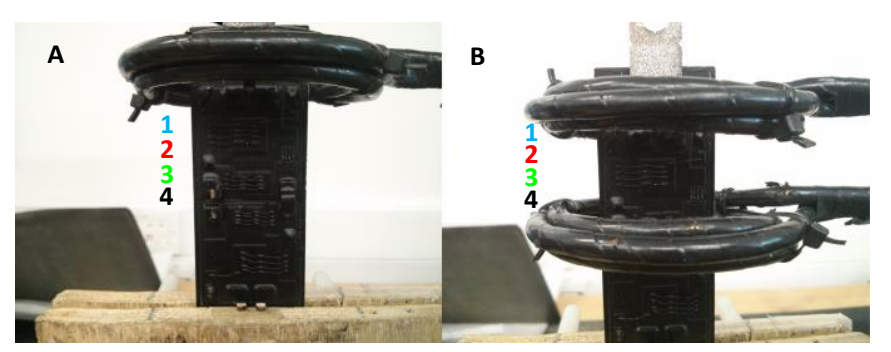

Fig. 7 (a) Circular coil and configuration, configuration

(b) Helmholtz coil and

A circular coil, shown in Fig. 7 (a), is simulated by the bottom coil loop of the Helmholtz coil constructed from a 6.35 $\mathrm{mm}$ high conductivity hollow copper tube. For the circular coil, the coil's circular plane is positioned to one side of the IGBT module; the IR camera then captures images of the bond wires. The coil excited NMF is then perpendicular to the wires.

As illustrated in Fig. 7 (b), Helmholtz coil excitation is applied after the Circular coil. The IR camera captures images through the gap between the top and bottom coil loops which provides a UMF within the coil. Before carrying out the experimental studies, simulations of the proposed technologies are necessary. Lifted bond wires were required to be incorporated into the model to enable the validation of the feasibility of bond cutting in a real IGBT module. Simulation models of induction heating and IGBT were built in the latest version of COMSOL (4.3 b).

\section{SimUlation MODELS AND RESUlTS}

COMSOL multiphysics is a software package for implementing finite-element analysis, solvers and simulations for various physics and engineering applications, especially coupled phenomena, or multiphysics. For simulation of induction heating, the software contains the electro-magnetic-heat process; therefore COMSOL has been used to study differences between NMF and UMF excitations of ECPT on simple IGBT models. The simulation work aims to include: (a), a comparison between the thermal patterns of wires under circular and Helmholtz coil excitations to study the influence of different magnetic field excitations on wire temperature distribution. (b), to study thermal patterns of healthy and lifted wires for fault identification, and (c), to validate the feasibility of ECPT for IGBT bond wires lift-off detection.

\section{A. Models Building}

To simplify the simulation model, one complex IGBT structure has been skeletonised to a simple geometric model. It includes an IGBT junction and a copper pad which is used for connecting external terminals, which are connected by four
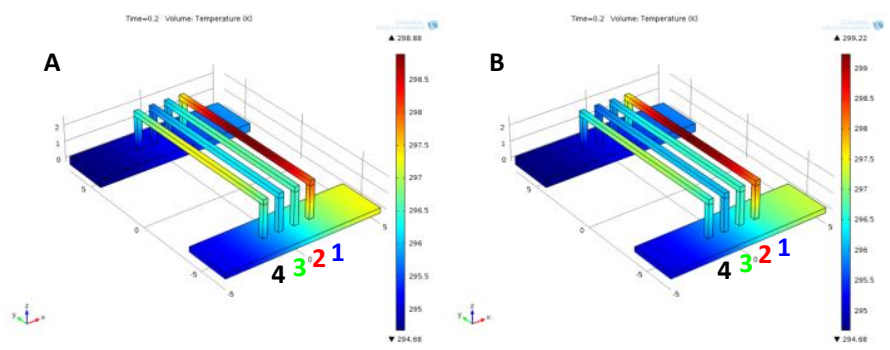
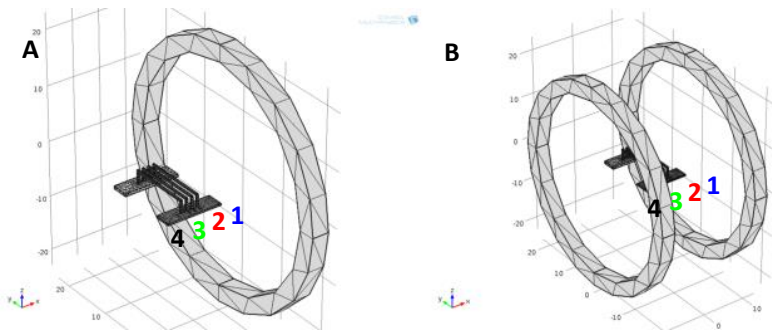

Fig. 8 Induction heating for IGBT model in COMSOL: (a) Circular coil excitation, (b) Helmholtz coil excitation.

aluminium bond wires. As introduced in section II, there is a 3 $\mu \mathrm{m}$ aluminium metallisation layer on the silicon die, which is used to provide better electrical conductivity and reduce the thermal coefficient mismatch between the bond wires and silicon die. Metallisation has rarely been mentioned in the literature except for the explanation of its functions and failure mechanisms related to it. Most researchers consider that the aluminium wires are directly bonded to the silicon die, either for simulations or experiments. However, for this paper, the level of induction heating in the metallisation layer is much larger than that in silicon die, as the electrical conductivity of silicon is $1.56 \times 10-3 \sigma(\mathrm{S} / \mathrm{m})$ at $20{ }^{\circ} \mathrm{C}$ and aluminium's is $3.5 \times 107 \sigma(\mathrm{S} / \mathrm{m})$ at $20{ }^{\circ} \mathrm{C}$. Hence, the junction has been considered to be aluminium metallisation in the simulation models. Fig. 8 shows the simplified model built in COMSOL. Each wire is numbered from right to left as 1 to 4 .

To compare the different induction heating on IGBT bond wires under NMF and UMF excitations, one IGBT module with four healthy wires and another one with one single lifted wire (wire 2) were built. Both of them were tested under of the two coil types, as shown in Fig. 7 (a) and Fig. 7 (b) respectively. The excitation boundary current density of the coils was set as $4.22 \times 107 \mathrm{~A} / \mathrm{m} 2$, which is the same as in the previous experiment. The diameter of coils' hollow tube is $3 \mathrm{~mm}$, which is used for water cooling; the radius of the coils is $20 \mathrm{~mm}$; and the distance between top and bottom coil loops of Helmholtz coil is $20 \mathrm{~mm}$. The induction heating includes a 200ms heating stage followed by a $800 \mathrm{~ms}$ cooling stage. To generalise and simulate a bond wire lift-off situation, one of the middle bond wires (wire 2) has been disconnected from the junction, with the gap between the wire wedge and junction being $0.01 \mathrm{~mm}$.

\section{B. Simulation Results}

Transient temperature distributions at the maximum heating time $(200 \mathrm{~ms})$ of the four models are shown in Fig. 9.

Fig. 9(c) indicates all of wires induced the same heating under the Helmholtz coil when they are of a healthy status, as the coil provides UMF excitation. Fig. 9(d) clearly shows that
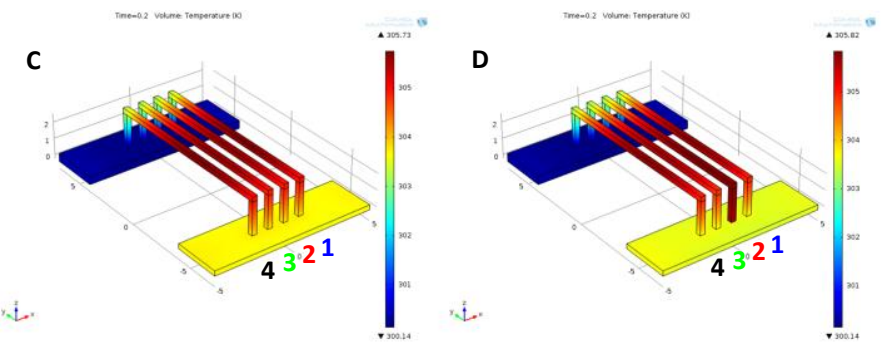

Fig. 9 Surface temperature images of simulation models at the maximum heating time 200 ms. (a) NMF excitation with well bonded wires. (b) NMF excitation with wire 2 defected. (c) UMF excitation with well bonded wires. (d) UMF excitation with wire 2 defected 

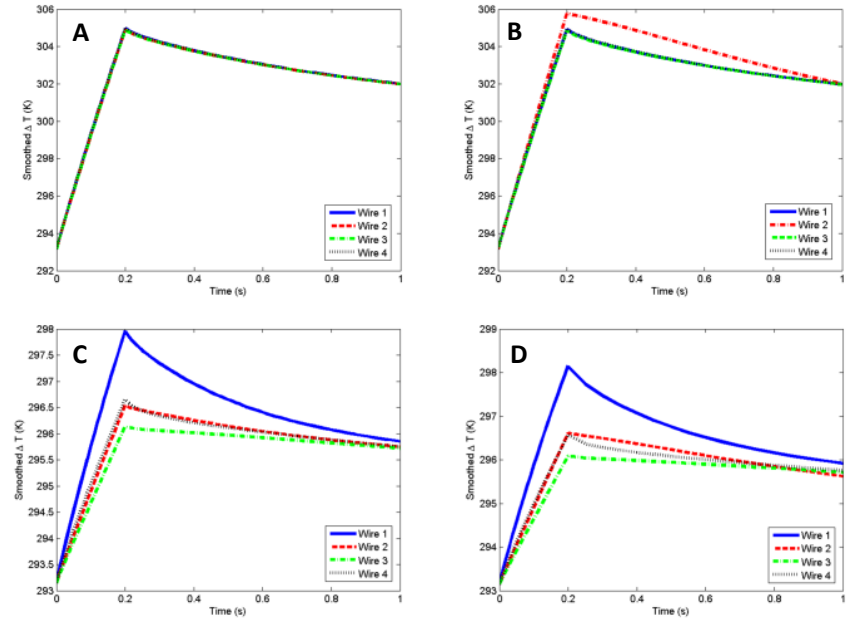

Fig. 10. Simulation results for temperature against time at the mid-point of the wires. (a) NMF excitation with well bonded wires. (b) NMF excitation with wire 2 defected. (c) UMF excitation with well bonded wires. (d) UMF excitation with wire 2 defected.

lifted wire 2 presents a higher temperature response than the other three health wires under Helmholtz coil excitation. However, the temperature responses are different across each of the four healthy wires and with lifted wire 2 when under circular coil excitation, as shown in Fig. 9(a) and Fig. 9(b). This is because they are situated in a NMF, which results in different densities of eddy currents being induced in each of them. Based on this premise, the lifted bond wire has no significant temperature difference related to its health state under circular coil excitation.

Fig. 10 shows simulation results for transient temperature against time at the central point of each wire in the related models in Fig. 9. All of the models present the same phenomenon, which is that the fringe wires indicate higher temperature responses than middle wires, due to electromagnetic proximity effects. As the coil provides a continuously varying magnetic field, it influences the distribution of the eddy currents flowing within the wires, by electromagnetic induction. Eddy currents in each wire induced by the magnetic field by the excitation coil create an associated alternating magnetic field around the wires. This alternating magnetic field then induces eddy currents in the adjacent wires, altering the overall distribution of current flowing through them. This result is that current is concentrated in the areas of the wires furthest away from nearby wires which are carrying current in the same direction.

The influence of electromagnetic proximity effect is illustrated vividly in Fig. 10 (a), when all of the wires are of a healthy status, heating in wires 1,2 and 3 are gradient reduced relative to their distance to excitation coil. However, heating in wire 4 is larger than in wires 2 and 3 even though it is furthest from the excitation coil. Due to the same reason, the heating patterns of wires under Helmholtz coil excitation indicate the same phenomenon, which is that the heating on the fringe wires is larger than for middle wires, but the differences are not significant enough, so they can be ignored in this case. As the wires are situated in a UMF, the heating on the wires is symmetrical so that fringe wires have same heating as the middle wires.

With circular coil excitation, the temperature distribution of the four healthy wires is unordered, however lifted wire 2 can still be identified in Fig. 10 (b). Since the lifted wire 2 has been disconnected from the aluminium metallisation, the heating generated in it cannot dissipate through the metallisation, as with the other healthy wires, so it shows a slightly larger heating process. For the same reason, after the heating stops, the cooling decay of lifted wire 2 is slower than it is in a healthy state at the start stage of cooling phase. At this stage, the heat diffusion is from wires to air, copper pad and aluminium metallisation. The health wires cool at a slower rate after the start stage of cooling phase due to heat transmission from aluminium metallisation.

For Helmholtz coil excitation, as shown in Fig. 10 (c), every healthy wire presents the same temperature response in both the heating and cooling phases as they do in a UMF, which results in similar amounts of eddy currents being induced in each of them. It is easy to identify the lifted wire 2 in Fig. 10 (d). It shows that the lifted wire 2 has a faster temperature rise in the heating phase. As with the circular coil excitation case, without a connection to aluminium metallisation, the eddy current loop in wire 2 does not flow through the metallisation, as such it enlarges the eddy current density of wire 2 and stops heat dissipation through to the metallisation. This results in larger heating being presented in lifted wire 2. During the cooling phase, lifted wire 2 shows a lower rate of temperature decay than other three healthy wires in the initial stage. This is because the heat of wire 2 is only transferred to the air and the copper pad while the heat of other wires was also transferred to the aluminium metallisation. After the initial stage, without heat transmission from the metallisation, the rate of temperature decay of wire 2 is higher than for other healthy wires.

To get a quicker meshing in the FEM model, there is a mismatch between the simulation model and a real world scenario. The copper pad has been adopted with a thickness of $500 \mu \mathrm{m}$ in the simulation instead of $300 \mu \mathrm{m}$ in the real IGBT module. Similarly, the thickness of aluminium metallisation is $300 \mu \mathrm{m}$ instead of $3 \mu \mathrm{m}$. Hence, the cooling stage of simulation may be different from it in the experimental results. As in reality metallisation cannot induce as much heat as in the simulation model, where the cooling rate of healthy wires will not be delayed by heat transmission from the metallisation.

The simulations proved the feasibility of applying ECPT to the detection of bond wire lift-off in an IGBT. Both NMF and UMF excitations enabled detection of the lifted wire, but the Helmholtz coil is more suitable in this case as it provides a more simple temperature distribution. One feature that can be extracted from the results above was the slopes of heating and cooling. This could be used as a feature to detect lifted bond wires. Based on the experiences of this simulation, experimental studies have been carried out in order to replicate the results of this section; experimental results and discussion are delivered in the next section.

\section{EXPERIMENTAL STUDIES}

The system introduced in section III is applied for the testing of an IGBT sample in a laboratory scenario. Two different kinds of excitations (circular and Helmholtz coils) have been used for producing NMF and UMF respectively. They are used for comparison of thermal patterns for status investigation of 

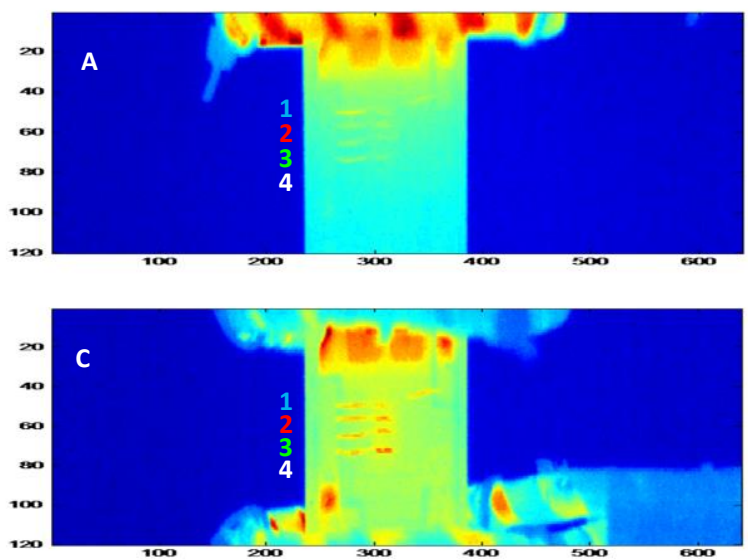
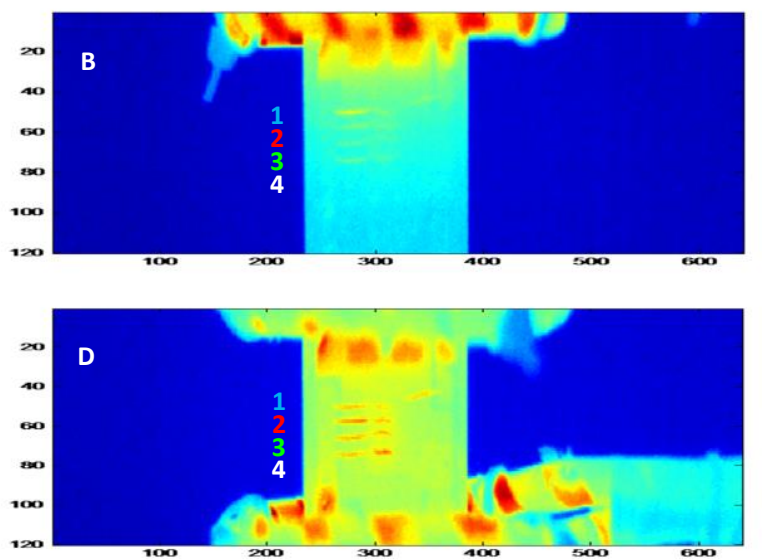

Fig.11 Infrared image at the maximum heating time 200 ms. (a) NMF excitation with well bonded wires. (b) NMF excitation with wire 2 defected. (c) UMF excitation with well bonded wires. (d) UMF excitation with wire 2 defected.

bond wires. Each excitation coil is tested using a sample in two conditions. The first condition is where all the wires are well bonded. The second condition is where wire 2 is lifted, and is to be considered a defect. A STARPOWER GD50HFU120C1S sample is used in the experimental tests; it is a molding type module, of specifications $1200 \mathrm{~V} / 50 \mathrm{~A}$ and 2 in one-package.

A $200 \mathrm{~ms}$ heating duration and $800 \mathrm{~ms}$ cooling period is selected for thermal image recording, which is long enough to elicit an observable heat pattern and cooling decay on bond wires. As configured in Fig. 7 (b), the sample is fixed and the Helmholtz coil can be moved up and down. In addition, normalisation of transient temperature change is applied, using division of the transient temperature by the temperature at maximum heating. The purpose of using normalisation is to reduce the influence of different lift-off distances between the coil and wires as well as to remove any influence from wire geometry [50].

ECPT was used to inspect the sample shown in Fig. 6. To compare the difference between NMF and UMF excitations, transient temperature responses of bond wires are compared. Fig.11 is the thermal images of four experiments at the maximum heating time of $200 \mathrm{~ms}$.

\section{A. Non-uniform magnetic field excitation}

The thermal images of the NMF excitation experiments, at the maximum heating time of $200 \mathrm{~ms}$, are shown in Fig.11 (a) and (b). They illustrate temperature distributions on bond wires with maximum heating. Fig.11 (a) shows the image of four health wires under NMF excitation. NMF induced different heating in wires, with wire 1 the closest to the coil; the magnetic field density is the largest at wire 1's position, which results in the highest heating being induced in wire 1 . Induction heating on wires 2, 3 and 4 are difficult to recognise by simple observation. Fig.11 (b) shows the image of wire 2 lifted under NMF excitation. As the heating induced in wires 2, 3 and 4 are relatively weak, it is hard to identify lifted wire 2 by visual inspection. The transient temperature responses at the central point of each wire under NMF excitation are shown in Fig.12 (a) and (b). It can be accepted that the density of the magnetic field decreases with increasing distance to the coil under NMF excitation. Consequently, the eddy currents induced across the area of wires 1 to 3 gradually decreases. Wire 4 has a higher density of eddy current than wires 2 and 3 due to influence of electromagnetic proximity effects. Therefore wire 4 presents a higher temperature response. After wire 2 was lifted, the heating process is faster than before due to there being no heat transfer to the IGBT junction (aluminium metallisation). Following the same logic, lifted wire 2 presents slower temperature decay during the cooling stage.

To reduce the influence of different lift-off distances between the coil and wires, as well as to remove the influence from wire geometry, normalisation of the transient temperature change has been applied. The normalisation results are shown in Fig.12 (c) and (d). Fig.12 (d) clearly shows wire 2 has a faster temperature rise in the heating phase and slower temperature dissipation in the cooling phase than those with a healthy state.

For this reason, in the case of NMF excitation employed to detect bond wire lift-off using ECPT, the failing wire can be identified after normalisation by its distinct thermal pattern, characterised by a faster temperature rise during the heating stage and a slower temperature dissipation during the cooling stage than those in a healthy state.

\section{B. Uniform magnetic field excitation}

Thermal images at the maximum heating time $200 \mathrm{~ms}$ of UMF excitation experiments are shown in Fig.11 (c) and Fig.11
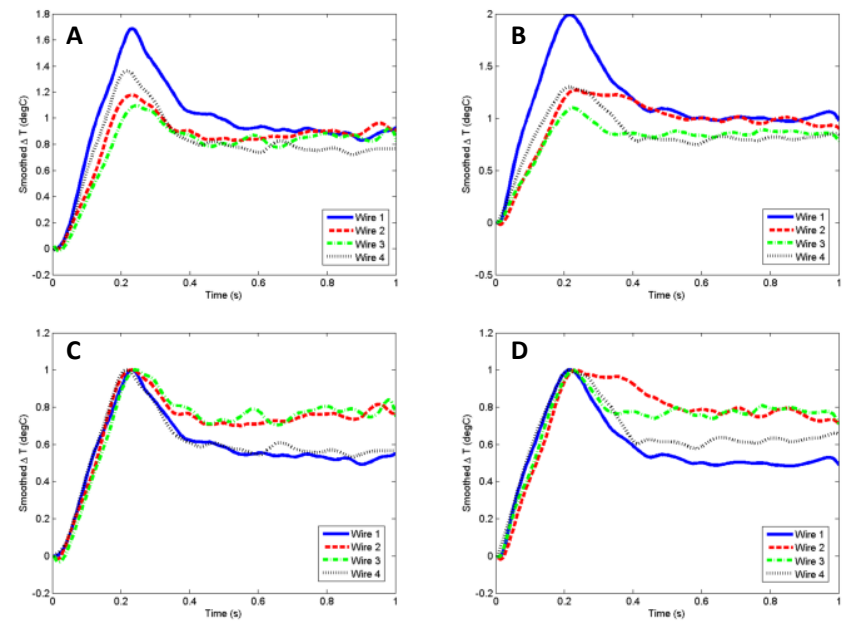

Fig.12 Experimental raw results and normalised results for temperature against time at the mid-point of the wires, under circular coil (NMF) excitation. (a) Experimental results of well bonded wires. (b) Experimental results of wire 2 defected. (c) Normalised results of well bonded wires. (d) Normalised results of wire 2 defected 
(d). They illustrate temperature distributions within and across the bond wires with maximum heating. Fig.11 (c) is an image of four healthy wires under UMF excitation. All of the wires present similar thermal responses. However lifted wire 2 presents a significant difference in thermal response to the other three healthy wires shown in Fig.11 (d). This is again due to there being no heat dissipation through the disconnected wire to the IGBT junction. Hence lifted wire 2 presents higher temperature response than other three healthy wires.

Fig.13 (a) and (b) indicate transient temperature change against time at the central point of each wire. Four well bonded wires present similar thermal responses under Helmholtz coil, shown in Fig.13 (a). Due to electromagnetic proximity effects, wires 2 and 3 have induced in themselves a relatively smaller heat than those in wires 1 and 4. Fig.13 (b) shows the transient responses when wire 2 has been lifted. It clearly shows that wire 2 has a higher rise in temperature during the heating stage and slower temperature dissipation in cooling stage the other three healthy wires.

For this reason, in the case of UMF excitation employed to detect bond wire lift-off using ECPT, the failure wire can be identified by both contrasting the distinct thermal patterns of the raw heating slopes and normalised results, where the failure wire has faster rise in temperature during the heating stage and slower temperature dissipation in the cooling stage than those when it is at healthy status.

The experimental results indicate that through analysis of the change in thermal patterns, both NMF and UMF excitations can be used for bond wire lift-off detection. Due to simple temperature distribution in bond wires, the UMF, excited by the Helmholtz coil, is more suitable for our application. Distinct slopes of temperature responses after normalisation can be used as feature to identify lifted wires. It shows the lifted wire has a temperature slope with faster heating and slower cooling periods than for healthy wires. Multiple-cycle excitations have been used in the experiments, which guarantee the repeatability of the test.

\section{CONCLUSION AND FUtURE WORK}

In this paper, both simulation and experimental studies for eddy current pulsed thermography system have been successfully applied for the detection of bond wire states in power electronic modules; namely IGBT samples. Both results of simulation and experimental works match each other. The method allows users to monitor the temperature distribution, which reflects eddy current distribution, across the multiple bond wires by using IR camera and allows detection of bond wire lift-off within a short time. Compared to traditional techniques for bond wire lift-off detection, ECPT offers non-contact inspection and is able to detect the status of all wires in IGBTs in a single pass. Repeatability of state detection of bond wires in IGBT modules using ECPT has been proven by testing another IGBT sample with another IR camera.

Two excitation coils: non-uniform and uniform magnetic field excitation, using circular and Helmholtz coils respectively have also been compared. The circular coil excitation provides a non-uniform magnetic field, which results in different eddy currents being induced in the bond wires. Because of the influence of proximity effects, the furthest wire from the coil
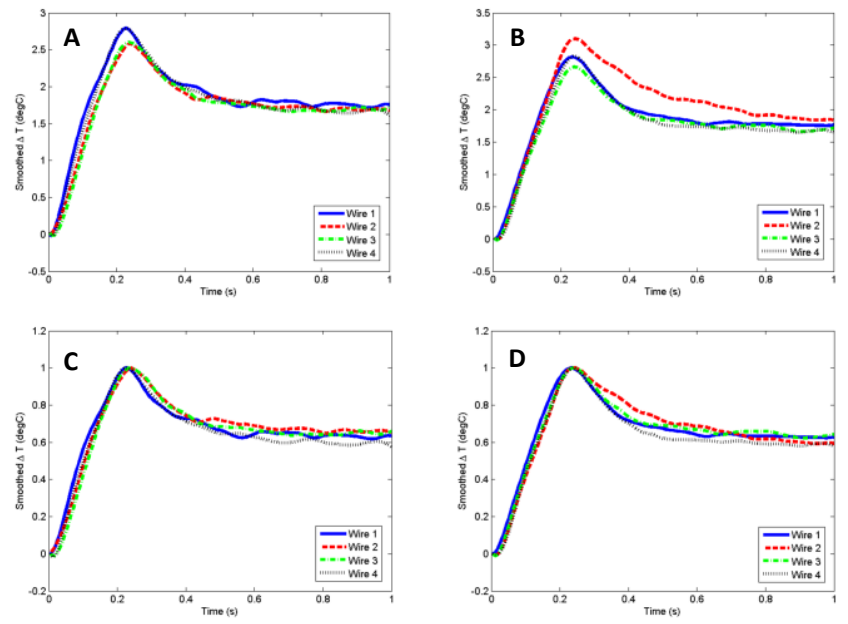

Fig.13 Experimental raw results and normalised results for temperature against time at the mid-point of the wires, under Helmholtz coil (UMF) excitation. (a) Experimental results of well bonded wires. (b) Experimental results of wire 2 defected. (c) Normalised results of well bonded wires. (d) Normalised results of wire 2 defected

has a higher temperature response than the middle wires. As such it causes difficulties when identifying defective wires. Under uniform magnetic field excitation, all healthy wires present similar thermal patterns. The influences of proximity effects also exist, but with a smaller impact, this results in higher levels of heat being induced in fringe wires than those in the middle. They present a symmetrical temperature distribution where the fringe wires present the same thermal responses, as the middle wires.

After normalisation of the transient thermal responses, lifted wires could be identified by their distinct temperature slope. This distinct temperature slope exhibited a faster temperature rise during the heating stage and a slower dissipation in the cooling stage.

Due to the simple temperature distribution of wires under uniform magnetic field excitation, the Helmholtz coil was found to be more suitable for the detection of bond wire lift-off, as such it will be employed in future studies in eddy current pulsed thermography systems.

The orientation of coils was found to be important, helping to achieve the best temperature contrast between the wires with and without defects. For the simulation studies, the junction die cannot be considered as silicon but aluminium metallisation.

To simplify model meshing and faster computation, the copper pad has been adopted with a thickness of $500 \mu \mathrm{m}$ instead of $300 \mu \mathrm{m}$ in the real module, $300 \mu \mathrm{m}$ sickness of aluminium metallisation has been adopted in the simulation instead of 3 $\mu \mathrm{m}$ in real test conditions. This resulted in larger heating being induced in the copper pad and aluminium metallisation which delayed the heat dissipation of the wires in the simulation.

Quantitative analysis on the distribution of eddy currents in bond wires will be carried out in the future work. Based on QNDE, bond wire health state, including material degradation and stress/strain, will also be further investigated. NDE means on bond layers and IGBT semiconductor dies will be investigated after these bond wire studies. SiC devices health monitoring using the ECPT will also be examined in future work. 


\section{ACKNOWLEDGEMENT}

The authors would like to thank Dr Bing Ji from Newcastle University for providing IGBT samples used in the paper. The work is partially funded by Europe Health Monitoring of Offshore Wind Farms (HEMOW) project.

\section{REFERENCES}

[1] B. K. Bose, "Power Electronics and Motor Drives Recent Progress and Perspective," Industrial Electronics, IEEE Transactions on, vol. 56, no. 2, pp. 581-588, 2009.

[2] Y. Shaoyong, A. Bryant, P. Mawby, X. Dawei, L. Ran, and P. Tavner, "An Industry-Based Survey of Reliability in Power Electronic Converters," Industry Applications, IEEE Transactions on, vol. 47, no. 3, pp. 1441-1451, 2011.

[3] M. Ciappa, and P. Malberti, "Plastic-strain of aluminium interconnections during pulsed operation of IGBT multichip modules," Quality and Reliability Engineering International, vol. 12, no. 4, pp. 297-303, 1996.

[4] S. Yang, D. Xiang, A. Bryant, P. Mawby, L. Ran, and P. Tavner, "Condition Monitoring for Device Reliability in Power Electronic Converters: A Review," Power Electronics, IEEE Transactions on, vol. 25, no. 11, pp. 2734-2752, 2010.

[5] M. Ciappa, "Selected failure mechanisms of modern power modules," Microelectronics Reliability, vol. 42, no. 4-5, pp. 653-667, 4//, 2002.

[6] Junhui Li, Deng Luhua, Ma Bangke, Wang Fuliang, Lei Han, “ Interfacial microstructures and thermodynamics of thermosonic $\mathrm{Cu}$-wire bonding," IEEE Electron Device Letters, vol. 32, no. 10, pp. 1433-1435, 2011.

[7] J. Li, L. Han, J. Duan, and J. Zhong, "Interface mechanism of ultrasonic flip chip bonding," Applied Physics Letters, vol. 90, no. 24, pp. 242902-242902-3, 2007.

[8] Li Junhui, Zhang Xiaolong, Liu Linggang, and Han Lei, "Interfacial characteristics and dynamic process of $\mathrm{Au}$-wire and $\mathrm{Cu}$-wire bonding \& overhang bonding in microelectronics packaging," IEEE/ASME Journal of Microelectromechanical Systems, vol. 22, no. 3, pp. 560-568, Jun, 2013.

[9] V. Smet, F. Forest, J. Huselstein, F. Richardeau, Z. Khatir, S. Lefebvre, and M. Berkani, "Ageing and Failure Modes of IGBT Modules in High-Temperature Power Cycling," Industrial Electronics, IEEE Transactions on, vol. 58, no. 10, pp. 4931-4941, 2011.

[10]P. Jacob, M. Held, P. Scacco, and W. Wuchen, "Reliability testing and analysis of IGBT power semiconductor modules." in Proc. IEE Colloq. IGBT Propulsion Drive, Apr. 25,1995, pp. 4/1-4/5.

[11]X. Dawei, R. Li, P. Tavner, A. Bryant, Y. Shaoyong, and P. Mawby, "Monitoring Solder Fatigue in a Power Module Using Case-Above-Ambient Temperature Rise," Industry Applications, IEEE Transactions on, vol. 47, no. 6, pp. 2578-2591, 2011.

[12] W. Kexin, D. Mingxing, X. Linlin, and L. Jian, "Study of Bonding Wire Failure Effects on External Measurable Signals of IGBT Module," Device and Materials Reliability, IEEE Transactions on, vol. PP, no. 99, pp. 1-1, 2012.

[13]H. Hui, and P. A. Mawby, "A Lifetime Estimation Technique for Voltage Source Inverters," Power Electronics, IEEE Transactions on, vol. 28, no. 8, pp. 4113-4119, 2013.

[14]X. Dawei, L. Ran, P. Tavner, Y. Shaoyong, A. Bryant, and P. Mawby, "Condition Monitoring Power Module Solder Fatigue Using Inverter Harmonic Identification," Power Electronics, IEEE Transactions on, vol. 27, no. 1, pp. 235-247, 2012.

[15]J. Wilson, G. Tian, I. Mukriz, and D. Almond, "PEC thermography for imaging multiple cracks from rolling contact fatigue," $N D T$ \& $E$ International, vol. 44, no. 6, pp. 505-512, 10, 2011.

[16]A. Yin, B. Gao, G. Yun Tian, W. L. Woo, and K. Li, "Physical interpretation and separation of eddy current pulsed thermography," Journal of Applied Physics, vol. 113, no. 6, pp. 064101-064101-8, 2013.

[17]H. Zhang, G. Tian, Y. He, and X. Zuo, "Defect depth effects in Pulsed Eddy Current thermography." Automation and Computing (ICAC), 2011 17th International Conference on, pp. 251-254.

[18]M. Mermet-Guyennet, "Reliability requirement for traction power converters," ECPEWorkshop 'Built-in Rel. Power Electron. Syst.'Toulouse, France, , Jun, 2008.

[19]A. Ahmed, Y. Shadrokh, L. Coulbeck, A. Castellazzi, and C. M. Johnson, "A closed-loop IGBT non-destructive tester," Microelectronics Reliability, vol. 52, no. 9-10, pp. 2358-2362, 9, 2012.
[20] M. A. Rodriguez, A. Claudio, D. Theilliol, and L. G. Vela, "A New Fault Detection Technique for IGBT Based on Gate Voltage Monitoring." in IEEE Power Electron. Spec. Conf., 2007, pp. 1001-1005.

[21] M. Ciappa, P. Malberti, W. Fichtner, P. Cova, L. Cattani, and F. Fantini, "Lifetime extrapolation for IGBT modules under realistic operation conditions," Microelectronics Reliability, vol. 39, no. 6-7, pp. 1131-1136, 6, 1999.

[22] X. Yali, C. Xu, Z. J. Shen, M. Chunting, W. Hongjie, and V. K. Garg, "Prognostic and Warning System for Power-Electronic Modules in Electric, Hybrid Electric, and Fuel-Cell Vehicles," Industrial Electronics, IEEE Transactions on, vol. 55, no. 6, pp. 2268-2276, 2008.

[23] N. Patil, J. Celaya, D. Das, K. Goebel, and M. Pecht, "Precursor Parameter Identification for Insulated Gate Bipolar Transistor (IGBT) Prognostics," Reliability, IEEE Transactions on, vol. 58, no. 2, pp. 271-276, 2009.

[24]M. Tounsi, A. Oukaour, B. Tala-Ighil, H. Gualous, B. Boudart, and D. Aissani, "Characterization of high-voltage IGBT module degradations under PWM power cycling test at high ambient temperature," Microelectronics Reliability, vol. 50, no. 9-11, pp. 1810-1814, 9, 2010.

[25]D. W. Brown, M. Abbas, A. Ginart, I. N. Ali, P. W. Kalgren, and G. J. Vachtsevanos, "Turn-Off Time as an Early Indicator of Insulated Gate Bipolar Transistor Latch-up," Power Electronics, IEEE Transactions on, vol. 27, no. 2, pp. 479-489, 2012.

[26] V. Smet, F. Forest, J. Huselstein, A. Rashed, and F. Richardeau, "Evaluation of Vce Monitoring as a Real-Time Method to Estimate Aging of Bond Wire-IGBT Modules Stressed by Power Cycling," Industrial Electronics, IEEE Transactions on, vol. 60, no. 7, pp. 2760-2770, 2013.

[27]Rodri, x, M. A. guez-Blanco, S. Claudio, x, A. nchez, D. Theilliol, V. Vela, x, L. G. s, T. Sibaja, x, P. n, Herna, x, G. ndez, x, L. lez, and J. Aguayo-Alquicira, "A Failure-Detection Strategy for IGBT Based on Gate-Voltage Behavior Applied to a Motor Drive System," Industrial Electronics, IEEE Transactions on, vol. 58, no. 5, pp. 1625-1633, 2011.

[28] L. Zhou, S. Zhou, and M. Xu, "Investigation of gate voltage oscillations in an IGBT module after partial bond wires lift-off," Microelectronics Reliability, vol. 53, no. 2, pp. 282-287, 2, 2013.

[29]Z. Shengqi, Z. Luowei, and S. Pengju, "Monitoring Potential Defects in an IGBT Module Based on Dynamic Changes of the Gate Current," Power Electronics, IEEE Transactions on, vol. 28, no. 3, pp. 1479-1487, 2013.

[30]P. Hofer-Noser, and N. Karrer, "Monitoring of paralleled IGBT/diode modules," Power Electronics, IEEE Transactions on, vol. 14, no. 3, pp. 438-444, 1999.

[31]B. Liu, D. Liu, Y. Tang, and M. Chen, "The Investigation on the Lifetime Prediction Model of IGBT Module," Energy Procedia, vol. 12, pp. 394-402, 2011.

[32]B. Farokhzad, P. Türkes, E. Wolfgang, and K. Goser, "Reliability indicators for lift-off of bond wires in IGBT power-modules," Microelectronics Reliability, vol. 36, no. 11-12, pp. 1863-1866, 11, 1996.

[33] O. Alatise, I. Kennedy, G. Petkos, and A. Koh, "Reliability of Repetitively Avalanched Wire-Bonded Low-Voltage Discrete Power Trench n-MOSFETs," Device and Materials Reliability, IEEE Transactions on, vol. 11, no. 1, pp. 157-163, 2011.

[34]J. M. Anderson, R. W. Cox, and J. Noppakunkajorn, "An on-line fault diagnosis method for power electronic drives." Electirc Ship Technologies Symposium (ESTS), 2011 IEEE;pp. 492-497.

[35]H. W. Sheng, F.; Tipton IV, C. W., "A Fault Detection and Protection Scheme for Three-Level DC-DC Converters Based on Monitoring Flying Capacitor Voltage," Power Electronics, IEEE Transactions on, vol. 27, no. 2, pp. 685-697, Feb. 2012.

[36]D. B. Jiang, R.; Wang, F.; Boroyevich, D., "Temperature-Dependent Characteristics of SiC Devices: Performance Evaluation and Loss Calculation," Power Electronics, IEEE Transactions on, vol. 27, no. 2, pp. 1013-1024, Feb. 2012.

[37] A. Ahmed, Y. Shadrokh, L. Coulbeck, A. Castellazzi, and C. M. Johnson, "A closed-loop IGBT non-destructive tester," Microelectronics Reliability, vol. 52, no. 9-10, pp. 2358-2362, 2012.

[38]Z. Lizheng, I. C. Ume, J. Gamalski, and K. P. Galuschki, "Detection of flip chip solder joint cracks using correlation coefficient and auto-comparison analyses of laser ultrasound signals," Components and Packaging Technologies, IEEE Transactions on, vol. 29, no. 1, pp. 13-19, 2006.

[39]Z. Lizheng, I. C. Ume, J. Gamalski, and K. P. Galuschki, "Study of Flip Chip Solder Joint Cracks Under Temperature Cycling Using a Laser Ultrasound Inspection System," Components and Packaging Technologies, IEEE Transactions on, vol. 32, no. 1, pp. 120-126, 2009.

[40]Y. Jin, and I. C. Ume, "Laser ultrasonic technique for evaluating solder bump defects in flip chip packages using modal and signal analysis 
methods," Ultrasonics, Ferroelectrics and Frequency Control, IEEE Transactions on, vol. 57, no. 4, pp. 920-932, 2010.

[41] J. Li, X. Zhang, L. Liu, L. Deng, and L. Han, "Effects of Ultrasonic Power and Time on Bonding Strength and Interfacial Atomic Diffusion During Thermosonic Flip\&Chip Bonding," Components, Packaging and Manufacturing Technology, IEEE Transactions on, vol. 2, no. 3, pp. 521-526, 2012.

[42]P. Godignon, X. Jorda, M. Vellvehi, X. Perpina, V. Banu, D. Lopez, J. Barbero, P. Brosselard, and S. Massetti, "SiC Schottky Diodes for Harsh Environment Space Applications," Industrial Electronics, IEEE Transactions on, vol. 58, no. 7, pp. 2582-2590, 2011.

[43] G. Breglio, A. Irace, E. Napoli, M. Riccio, P. Spirito, K. Hamada, T. Nishijima, and T. Ueta, "Detection of localized UIS failure on IGBTs with the aid of lock-in thermography," Microelectronics Reliability, vol. 48, no. 8-9, pp. 1432-1434, 8, 2008.

[44] T. Kociniewski, J. Moussodji, Z. Khatir, M. Berkani, S. Lefebvre, and S. Azzopardi, "New Investigation Possibilities on Forward Biased Power Devices Using Cross Sections," Electron Device Letters, IEEE, vol. 33, no. 4, pp. 576-578, 2012.

[45]D. P. Almond, B. Weekes, T. Li, S. G. Pickering, E. Kostson, J. Wilson, G. Y. Tian, S. Dixon, and S. Burrows, "Thermographic techniques for the detection of cracks in metallic components," Insight: Non-Destructive Testing and Condition Monitoring, vol. 53, no. 11, pp. 614-620, 2011.

[46] M. Noethen, K. J. Wolter, and N. Meyendorf, "Surface crack detection in ferritic and austenitic steel components using inductive heated thermography." in Proc. 33rd Int. Spring Seminar Electron. Technol. (ISSE), 2010, pp. 249-254.

[47]N. Biju, N. Ganesan, C. V. Krishnamurthy, and K. Balasubramaniam, "Simultaneous Estimation of Electrical and Thermal Properties of Isotropic Material from the Tone-Burst Eddy Current Thermography (TBET) Time\&Temperature Data," Magnetics, IEEE Transactions on, vol. 47, no. 9, pp. 2213-2219, 2011.

[48]C. Liang, and T. Gui Yun, "Surface Crack Detection for Carbon Fiber Reinforced Plastic (CFRP) Materials Using Pulsed Eddy Current Thermography," Sensors Journal, IEEE, vol. 11, no. 12, pp. 3261-3268, 2011.

[49]C. Liang, and T. Gui Yan, "Transient Thermal Behavior of Eddy-Current Pulsed Thermography for Nondestructive Evaluation of Composites," Instrumentation and Measurement, IEEE Transactions on, vol. 62, no. 5, pp. 1215-1222, 2013.

[50]L. Wei, C. Guoming, Y. Xiaokang, Z. Chuanrong, and L. Tao, "Analysis of the lift-off effect of a U-shaped ACFM system," NDT \& E International, vol. 53, no. 0, pp. 31-35, 1, 2013.

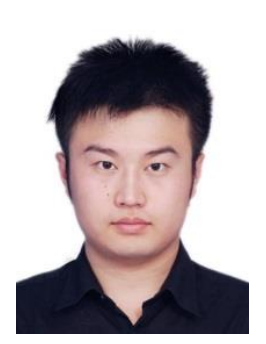

Kongjing Li (M'13) received the BEng degree in electronic engineering from the University of Hull, Hull, U.K., in 2010 and the MSc degree in electrical technology for sustainable and renewable energy systems from the University of Nottingham, Nottingham, U.K., in 2011. He is currently pursuing the Ph.D. degree in electrical and electronics engineering at Newcastle University, Newcastle upon Tyne, U.K. His research interests include NDT\&E for reliability and health monitoring of power electronic systems.

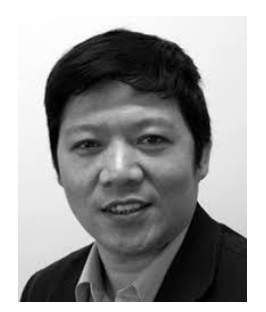

Gui Yun Tian (M'01-SM'03) received the B.Sc. degree in metrology and instrumentation and the M.Sc. degree in precision engineering from the University of Sichuan, Chengdu, China, in 1985 and 1988, respectively, and the Ph.D. degree from the University of Derby, Derby, U.K., in 1998. He was a Lecturer, Senior Lecturer, Reader, Professor, and Head of the Group of Systems Engineering, University of Huddersfield, Huddersfield, U.K., from 2000 to 2006, Since 2007, he has been based at Newcastle University, Newcastle upon Tyne, U.K., where he has been the Chair Professor in sensor technologies. Currently, he is also with the School of Automation Engineering, University of Electronic Science and Technology of China, Chengdu, China. $\mathrm{He}$ has coordinated several research projects from the Engineering and Physical Sciences Research Council, Royal Academy of Engineering and FP7, on top of this he also has good collaboration with leading industrial companies, such as Airbus, Rolls Royce, BP, nPower, and TWI.

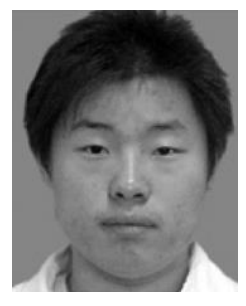

Liang Cheng was born in Beijing, China, in 1985. He received the B.Sc. degree in electronics from Peking University, Beijing, China, in 2007, and the M.Sc. degree in communications and signal processing from Imperial College London, London, U.K., in 2008 and the Ph.D. degree from the Newcastle University, Newcastle upon Tyne, U.K., in 2013. He is currently as research assistance at Newcastle University. His research interests include electromagnetic NDT\&E for composites and metallic materials used in renewable energy.

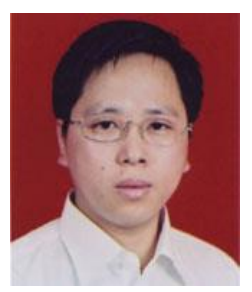

Aijun Yin (M'13) received his BS degree in mechatronics engineering from Chongqing University in Chongqing, China, in 2001. He earned his MS and PhD degrees in 2003 and 2006, respectively. He is currently an associate professor in College of Mechanical Engineering of Chongqing University. His research interests include machine vision and image processing, intelligent test and instruments, non-destructive testing and evaluation, modern signal analysis and processing, and fault detection and diagnosis.

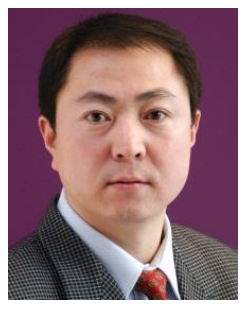

Wenping Cao (M'05-SM'11) received the BEng in electrical engineering from Beijing Jiaotong University, Beijing, China, in 1991; and the Ph.D. degree in electrical machines and drives from the University of Nottingham, Nottingham, U.K., in 2004. He is currently a Senior Lecturer with Queen's University Belfast, Belfast, U.K. His research interests are in fault analysis and condition monitoring of electric machines, drives and power electronics.

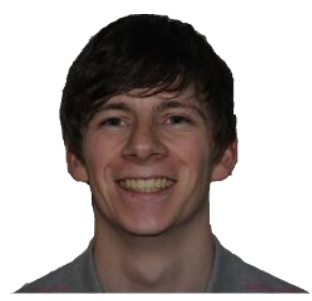

Stuart Crichton is currently a $\mathrm{PhD}$ candidate at the School of Electrical and Electronic Engineering and the Institute of Neuroscience at Newcastle University. He has published, and has interests in, the colour constancy, colour science and psychophysics fields and is interested in bridging the gap between human colour vision, perception and computer vision. Within the computer vision field he has interests in both colour image processing and more general image processing algorithms. 